\title{
Solar polarimetry in the near UV with the Zurich Imaging Polarimeter ZIMPOL II
}

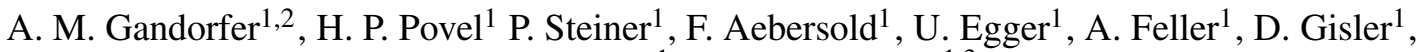 \\ S. Hagenbuch ${ }^{1}$, and J. O. Stenflo ${ }^{1,3}$ \\ 1 Institute for Astronomy, ETH Zentrum, 8092 Zurich, Switzerland \\ e-mail: gandorfer@linmpi.mpg.de \\ 2 Max-Planck-Institut für Sonnensystemforschung ${ }^{\star \star}$, 37191 Katlenburg-Lindau, Germany \\ 3 Faculty of Mathematics \& Science, University of Zurich, Switzerland \\ Received 12 February 2004 / Accepted 14 April 2004
}

\begin{abstract}
We describe an imaging polarimeter for high sensitivity measurements of solar polarisation signals in the wavelength range from $300 \mathrm{~nm}$ to $1 \mu \mathrm{m}$. At higher wavelengths the system is limited by the wavelength cut-off of the silicon CCD sensor used. To the blue the limitation arises from the atmospheric cut-off around $310 \mathrm{~nm}$. The complete system is a modified version of the Zurich Imaging Polarimeter ZIMPOL II which has been equipped with a special CCD sensor. The CCD combines for the first time a so-called open electrode structure with on-chip demodulation. The concept as well as the detailed design of the instrument are presented. Examples of observations are shown and interpreted in order to experimentally evaluate the performance of the system.
\end{abstract}

Key words. instrumentation: polarimeters - instrumentation: detectors - techniques: polarimetric - polarization Sun: magnetic fields - ultraviolet: general

\section{Introduction}

Highly sensitive observations of solar polarisation have revealed new insights into a variety of physical phenomena in the solar atmosphere. While the observation and interpretation of polarisation due to the Zeeman effect has been used for a long time and has been developed into an ever more sophisticated diagnostic tool for solar surface magnetism, the observation of scattering polarisation has long suffered from a lack of polarimetric sensitivity in combination with the intrinsic weakness of the observable polarisation effects.

During recent years the situation has dramatically changed with the advent of new imaging polarimeters which allow for detailed investigation of non magnetic line polarisation that is caused by scattering in the upper layers of the photosphere and in the chromosphere. This polarisation has a rich spectral structuring due to different physical mechanisms contributing to the linear polarisation that is observed near the solar limb where the observational geometry is favorable to detect the scattered radiation. The spectral distribution of the scattering polarisation at the solar limb has therefore been refered to as the "second solar spectrum" (Ivanov 1991).

* All appendices are only available in electronic form at http://www . edpsciences.org

$\star \star$ Formerly known as Max-Planck-Institut für Aeronomie.
While the strongest polarising lines of the second solar spectrum had been detected already in the seventies (Stenflo 1974; Wiehr 1975, 1978; Stenflo et al. 1980), the majority of the polarisation effects could only be addressed with the highly sensitive Zurich Imaging Polarimeters ZIMPOL I and ZIMPOL II (Povel 1995, 2001; Gandorfer \& Povel 1997; Stenflo \& Keller 1996, 1997; Stenflo et al. 1998, 2000a,b, 2001; Gandorfer 2000, 2002).

In the ZIMPOL concept the two major systematic error sources are eliminated: while the infiltration of seeing-induced cross talk is avoided by ultrafast polarisation modulation in the tens of $\mathrm{kHz}$ range, the noise contributions by the gain table or flat field effects are ruled out by a special CCD sensor that is itself used as part of a synchronous demodulator (Povel 1995, 2001; Povel et al. 1990, 1994; Gandorfer \& Povel 1997). With this concept, which will be described in more detail below, the sensitivity of the polarisation analysis is dominated by the shot noise of the collected photoelectrons.

The former versions of the ZIMPOL polarimeter were based on a three phase buried channel silicon CCD which is front illuminated. This device is sensitive only above $450 \mathrm{~nm}$ since the shorter wavelengths are absorbed in the polysilicon electrodes and thus cannot penetrate into the photosensitive substrate.

This sets a limit to our former observations as we were not able to address the most promising wavelength region of the second solar spectrum below $450 \mathrm{~nm}$ : as the anisotropy of 
the illumination which is the fundamental polarisation source steeply increases when going to shorter wavelengths, the amplitudes of the polarisation signals become larger in the blue part of the spectrum. Also the number density of resonance lines with high intrinsic polarisabilities is higher at shorter wavelengths. For this reason there is urgent need for a highly sensitive polarimetric system working in this promising part of the solar spectrum. Although the beam-splitter system of the Locarno observatory has been used with great success in recent years (Bianda et al. 1998a,b, 1999a) we felt that the enormous advantages of the ZIMPOL concept should be exploited, and the concept should be expanded to the near ultraviolet part of solar radiation.

\section{Instrument design}

In the following section we will concentrate on the basic concepts of the instrument as well as its main components. For details on the individual design solutions we refer to the Appendix.

\subsection{Basic concept}

In an astronomical polarimeter, polarisation of light is transformed into intensity modulation. Due to seeing fluctuations, guiding errors and instrument instabilities the intensity fluctuates in a frequency range between several $10 \mathrm{mHz}$ and several $100 \mathrm{~Hz}$. The induced noise is supressed by modulating the signal at a frequency above this limit. Commonly used polarisation modulators are electro-optical devices like Pockels cells, ferro-electric liquid crystals (FLC) and photo-elastic modulators (PEM). PEMs are very convenient to use because they are stable, have high optical quality and are easily tunable over a wide useful wavelength range. Since they are operated at their mechanical resonance, the modulation frequency is rather high as opposed to FLCs and Pockels cells which can be driven in a wide frequency range up to several $\mathrm{kHz}$. Standard PEMs oscillate at several $10 \mathrm{kHz}$ depending on the size of the modulator.

With charge coupled devices (CCD), which can be read out at video frequency or multiples of it (multi-port arrays), synchronous demodulation of intensity signals in the $100 \mathrm{~Hz}$ range is possible. However, at low photon flux, associated with high spatial and/or spectral resolution, at the high pixel clock frequency (typically $10 \mathrm{MHz}$ ) needed to obtain a high frame rate, the readout noise becomes comparable to the shot noise of the charge accumulated per pixel during one frame period. The high modulation frequency of PEMs is not compatible with the frame readout frequency achievable with CCDs of reasonable size.

This problem has been solved with the Zurich Imaging Polarimeters ZIMPOL I (Povel 1995; Povel et al. 1990, 1994) and later with ZIMPOL II (Stenflo et al. 1992; Gandorfer \& Povel 1997) by using masked CCDs. With ZIMPOL I every second row of the CCD is covered by an opaque mask. The rows screened from light serve as temporary buffers, which allow the device to separate the charges generated at two different phases of the modulation, by shifting the charges back and forth in the vertical (column) direction, synchronously to the modulation. The frame is read out after integration over many modulation cycles. The demodulated signal is calculated offline as the relative difference between two corresponding rows. If the vertical (line) transfer time can be made sufficiently short, synchronous demodulation of intensity modulations in the $10 \mathrm{kHz}$ range with CCDs becomes possible. The most remarkable advantages of this technique are: (1) independence of the responsivity of individual pixels, because both polarisation states are detected with the same pixel; (2) combination of fast ( $\geq 1 \mathrm{kHz}$ ) polarisation modulators with CCDs; (3) integration over many modulation cycles without readout of the frame; (4) simple and compact setup.

The ZIMPOL I CCD can demodulate only one signal at a given frequency and phase. It cannot simultaneously detect signals at several frequencies and/or phases, and therefore is sensitive to only one polarisation parameter. An improved system, capable of recording simultaneously all Stokes parameters has been introduced with ZIMPOL II, where the frequency and phase detecting capabilities of the CCD demodulator have been extended by increasing the number of buffer rows. With 3 covered buffer rows and one uncovered row the modulation cycle can be sampled at 4 intervals by vertically shifting the frame synchronously to the modulation. If the modulation pattern is selected appropriately, 4 modulation parameters corresponding to the 4 Stokes parameters can be recorded simultaneously with a single CCD (Stenflo et al. 1992; Gandorfer 1999a,b).

\subsection{Polarisation modulation}

To avoid seeing induced errors, a fast polarisation modulation scheme with modulation frequencies in the $\mathrm{kHz}$ range is necessary. In the visible part of the spectrum a full vector modulation scheme based on ferroelectric liquid crystal modulators can be used (Gandorfer 1999a). Although the useful wavelength interval of a modulation system based on these chromatic devices may be drastically enhanced by careful optimization of the overall configuration (Gisler 2004), the degradation of the organic liquid crystal substrate at wavelengths below $400 \mathrm{~nm}$ inhibits their use in the UV. The UV sensitive version of the ZIMPOL II polarimeter therefore uses ultrafast polarisation modulation based on a photoelastic modulator. Details on the modulation scheme can be found in Appendix B.

\section{3. $C C D$ image sensor}

For fast on-chip demodulation the CCD has to meet the following requirements: (1) bidirectional vertical charge transfer (3-phase structure); (2) high and symmetrical vertical charge transfer efficiency (buried channel); (3) fast vertical transfer (low image area electrode impedance).

ZIMPOL I and II are used in a wavelength range mostly limited by the response of the CCD sensor, with a maximum at about $700 \mathrm{~nm}$, less than $1 \%$ efficiency at $400 \mathrm{~nm}$ and virtually no response below $400 \mathrm{~nm}$. The low quantum efficiency (QE) at short wavelengths is due to the absorption of the incident light by the poly-silicon electrode structure on the front-side surface of the CCD associated with multi-phase technology 
(absorption depth in $\mathrm{Si}: \approx 3 \mathrm{~nm}$ at $\lambda=250 \mathrm{~nm}$, and $\approx 200 \mathrm{~nm}$ at $\lambda=400 \mathrm{~nm}$ ). Further reduction of $\mathrm{QE}$ is due to the increasing reflectivity of the electrode layer with decreasing wavelength $(70 \%$ at $250 \mathrm{~nm})$. To improve the response of CCDs below $400 \mathrm{~nm}$ several methods are known: (1) phosphor coating; (2) back-side illumination; and (3) open electrode structure. In an open electrode CCD the poly-silicon gate structure, which absorbs the UV radiation, is partly removed so that it leaves part of the pixel area uncovered. The first method cannot be used with masked CCDs due to excessive stray light in the screened pixel rows, and poor stability of the coating. The second method is expected to give good results, if the difficult alignment and mounting of the mask on the backside of the thinned chip with the required submicron precision could be managed. We have chosen the third method because it is less risky than the second one. However, it is more expensive since it requires a partial re-design of an existing $\mathrm{CCD}$, or even a new design.

We decided to modify a Marconi CCD55-20 $(2 \times 576(V) \times$ $770(H)$ pixels, $22.5 \mu \mathrm{m} \times 22.5 \mu \mathrm{m})$, which is an improved successor of CCD05-20 used with ZIMPOL II. To provide the sensor with open poly-silicon electrodes Marconi needed to redesign a number of masks and manufacture a part batch of devices.

A detailed description of the CCD sensor with "open electrode structure" can be found in Appendix C. Appendix D describes the CCD camera and its associated front-end electronics. A system overview with block diagram is provided in Appendix A. Step motor controller description and an overview of the Hardware Control Network and a detailed summary of the software concept and implementation may be found in Appendices E, F, G, respectively.

\section{Observational technique and calibration}

ZIMPOL II is a pure polarimeter, which can be used in combination with spectrographs and filters of any kind. Wavelength discrimination is necessary to unveil the polarisation signals, which in most cases show a strong wavelength dependence.

Since the ZIMPOL polarimeter is a versatile instrument it can be attached to most existing solar telescopes. Its primary application is spectropolarimetry at high spectral resolution, but it has been also proven a powerful instrument in combination with filtergraphs.

While examples of these different observational methods are described in Sect. 4, we refer to Appendices $\mathrm{H}$, I, and $\mathrm{J}$ for a discussion of details regarding the attachment of the instrument to the telescope/spectrograph and the calibration/observing procedure.

\section{Examples of observations}

Solar polarisation is caused by two different physical mechanisms. While magnetic fields may lead to observable circular and linear polarisation in magnetically sensitive spectral lines by shifting the energy levels of the atom (Zeeman effect), scattering gives rise to linear polarisation, both in the continuous and the line spectrum. This polarisation can be altered under the influence of magnetic fields; this effect is referred to as the Hanle effect. While Zeeman diagnostics has matured to a standard diagnostic technique in solar magnetic field diagnostics, scattering (Hanle) polarimetry is still at the starting point of becoming a diagnostic tool. The spectral regions, in which both techniques should be applied, are found on opposite sides of the visible solar spectrum: for a given magnetic field the Zeeman splitting scales with the square of the wavelength of the line, thus the natural observing ground for Zeeman measurements is the infrared part of the spectrum. Scattering becomes more and more pronounced for shorter wavelengths, such that Hanle diagnostics work best in the UV.

In this paper we show examples of observations that have been made with the UV sensitive version of ZIMPOL II. A number of different observing tasks has already been adressed during recent years. Here we will list three particularly interesting applications of the instrument to show the universality of the system.

\subsection{High sensitivity polarimetry of the solar limb}

Due to its high polarimetric sensitivity and wavelength tunability, ZIMPOL II is optimally suited for observations of scattering polarisation at the solar limb. Exploring this "second solar spectrum" requires high polarimetric sensitivity for the linear polarisation in combination with very high spectral resolution $(\geq 250000)$ in order to resolve the line profiles. Since without magnetic fields the scattering polarisation is a purely geometrical effect, which depends only on wavelength and position on the Sun, we can sacrifice spatial resolution for the sake of polarimetric accuracy. If the spectrograph entrance slit is placed parallel to the solar limb, then we can average along the spatial direction, which increases the photometric (and thus the polarimetric) signal-to-noise ratio by one order of magnitude. The resulting one-dimensional spectrum has been recorded systematically with ZIMPOL II between $3165 \AA$ and $6995 \AA$. While the region above $4625 \AA$ could already be explored with the normal version of ZIMPOL II at the IRSOL facility in Locarno (Gandorfer 2000), the shorter wavelengths were only adressable with the new system described in this paper. The wavelength region from $3910 \AA$ to $4630 \AA$ has also been recorded at IRSOL (Gandorfer 2002) while the shortest wavelengths down to $3165 \AA$ could only be registered at the $1.5 \mathrm{~m}$ McMath-Pierce telescope of NSO (Kitt Peak) (Gandorfer 2004). As an example of this type of observation a detailed view is presented in Fig. 1.

\subsection{Spatial/spectral signatures of the Hanle effect}

Strongly polarising spectral lines can be investigated with higher spatial resolution, since it is not necessary to spatially average the polarimetric data to increase the signal-to-noise ratio. Most of these strongly polarising lines occur in the UV part of the solar spectrum and are therefore adressable with the ZIMPOL II instrument. Observations of scattering polarisation in active regions reveal local influences of magnetic fields on the scattering polarisation. Polarimetry in UV spectral 


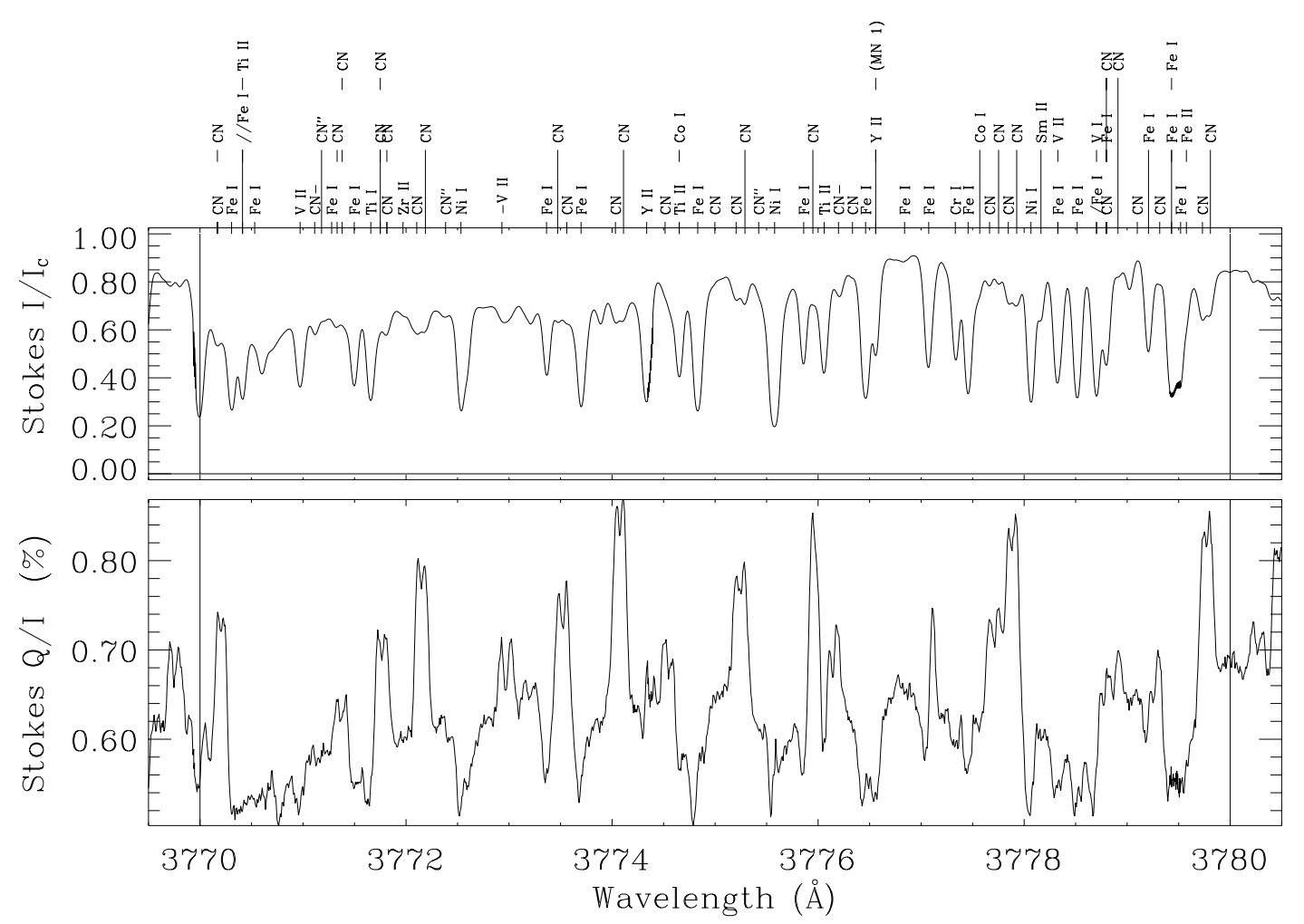

Fig. 1. Detail of the Fraunhofer spectrum (top panel) and the second solar spectrum (lower panel) around $377 \mathrm{~nm}$. In the fractional polarisation many signals of the $\mathrm{CN}$ molecule are visible, providing complementary information in addition to normal spectroscopy of the solar atmosphere.

lines is therefore a key observational technique for our understanding of chromospheric magnetism. A spectral line that has proven to be particularly suited for this purpose is the Ca I line around $4227 \AA$. While having been used earlier for magnetic field diagnostics (Bianda et al. 1999a,b), ZIMPOL II now allows investigations with much higher spatial resolution while even increasing polarimetric sensitivity (Bianda et al. 2003). An example of such an observation is shown in Fig. 2. The measurement of the complete Stokes vector in the spectral region around the strong $\mathrm{Ca}$ I line around $4227 \AA$ has been recorded with the ZIMPOL II polarimeter at the vertical spectrograph of the $1.5 \mathrm{~m}$ McMath-Pierce telescope of NSO (Kitt Peak) in March 2002. Observations like this one reveal complicated polarisation effects of both the Hanle and the Zeeman effects and are therefore of particular interest for the understanding of chromospheric magnetic fields.

\subsection{Topology of scattering polarisation}

Spatial mapping of the scattering polarisation and the signatures of the Hanle and Zeeman effects requires imaging polarimetry with narrowband filters. Observations of this kind can in principle be done with very high spatial resolution. For first tests ZIMPOL II was attached to the New Swedish Solar Telescope. Regions of different magnetic activity near the solar limb have been imaged through a $1 \AA$ bandpass interference filter centered at the core of the Ca II K line around $3933 \AA$. In contrast to the $\mathrm{Ca}$ II $\mathrm{H}$ line around $3968 \AA$ the $\mathrm{K}$ line exhibits strong resonance polarisation (cf. the atlas of Gandorfer 2002). Both lines, however, are sensitive to Zeeman effect
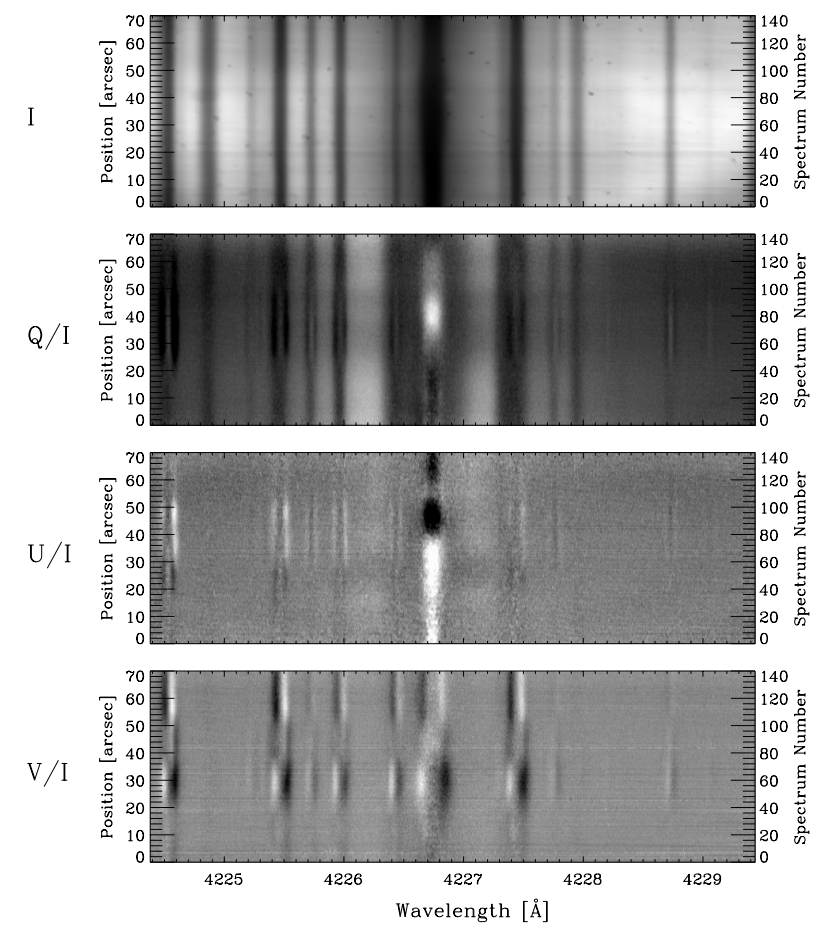

Fig. 2. Images of the four Stokes parameters in the spectral/spatial domain. The spectrograph entrance slit of the $1.5 \mathrm{~m}$ McMath-Pierce solar telescope has been set parallel to the solar limb at a distance of 10 arcsec inside the visible solar limb and covers 70 arcsec. The spectral field of view is centered on a line of neutral calcium. White and black correspond to $\pm 1 \times 10^{-2}$ in Stokes $Q / I$ and $V / I$, and to $\pm 4 \times 10^{-3}$ in Stokes $U / I$. 
Ca II K line core

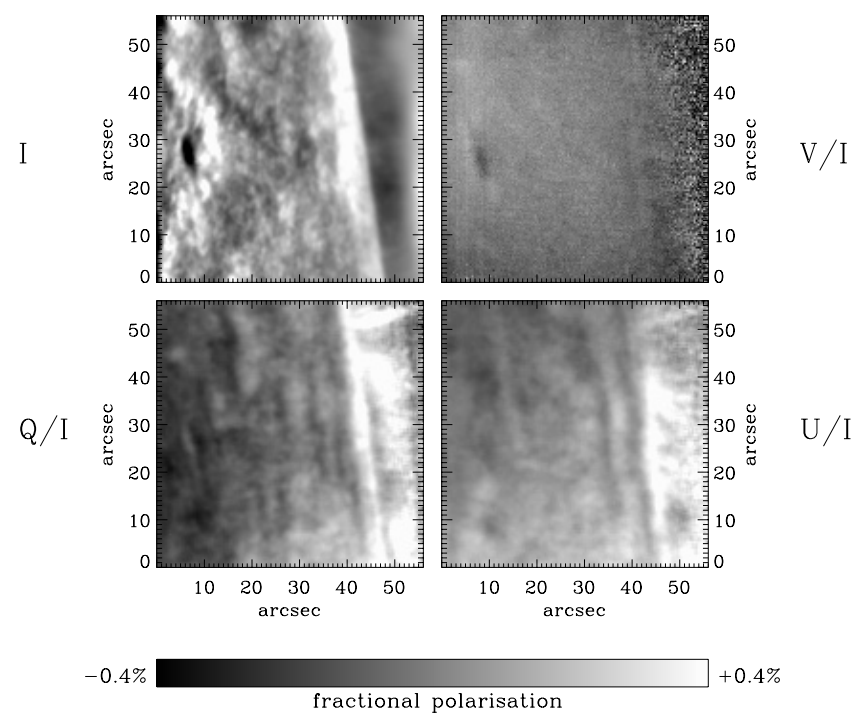

Fig. 3. Images of the four Stokes parameters in the spatial domain, recorded with ZIMPOL II at NSST on La Palma in October 2003, using a $1 \AA$ Ailter centered on the Ca II K line. The contrast in the intensity image has been enhanced by subtraction of the general center-to-limb variation. The gray scale of the fractional polarisation images is calibrated at the bottom of the picture.

(Martinez Pillet et al. 1990). Since the transmission band is fairly wide, Zeeman polarisation signals are expected to be below the detection limit, due to cancellation effects. The linear polarisation caused by scattering, as well as its modification by local weak magnetic fields give, however, detectable linear polarisation as can be seen in Fig. 3 not only in a narrow limb zone but also above the visible solar limb in spicules, as well as in regions corresponding to network boundaries. While circular polarisation due to the Zeeman effect is not detected due to spectral smearing, signatures of Hanle rotation are prominent in the limb zone as well as in the observed spicules. Observations of this type harbour potential for diagnostics of chromospheric magnetic fields.

\section{Discussion}

In the present paper we explained the working principle of the UV sensitive version of the Zurich Imaging Polarimeter ZIMPOL II. We also showed examples of observations that demonstrate the capabilities of the instrument. In the following we intend to critically review the actual system after having used the instrument for scientific observations. An outlook for further development steps concludes this paper.

\subsection{Characterisation of the instrument}

In solar polarimetry we face severe trade-offs between the various resolutions (spatial, spectral, temporal and polarimetric sensitivity) due to the limited photon flux. It is therefore essential to chose the observing procedure or even the instrumentation to optimally address a given scientific question. Thus we cannot directly compare the value of an instrument without selecting a distinct set of specifications. In the following we will go through the various resolutions individually and critically regard the performance of the ZIMPOL polarimeter.

\subsubsection{Polarimetric sensitivity}

As explained at the beginning of this paper the main noise sources in polarimetry are ruled out in ZIMPOL thanks to its working principles. The achievable noise levels are consistent with the statistics of accumulated photo-electrons. This means that in a single frame a noise level of $2 \times 10^{-3}$ of the intensity is reached. By frame averaging, this value can be suppressed to values around $2 \times 10^{-5}$. Evidently for such noise levels spatial and temporal resolutions must be largely sacrificed to gain sufficient signal.

\subsubsection{Spectral resolution}

ZIMPOL is a pure polarimeter that can be used in combination with any kind of wavelength discriminator. Since the modulation scheme is strictly monochromatic, ZIMPOL II is best used in observations with high to highest spectral resolution in a very limited spectral interval. To avoid calibration errors the maximum simultanously observed bandwidth should not exceed $1 \mathrm{~nm}$. On the other hand the modulator is wavelength tunable, giving the system the unique capability to be used at any wavelength between $300 \mathrm{~nm}$ and $1 \mu \mathrm{m}$.

\subsubsection{Spatial resolution}

Spatial resolution is a clear drawback of the actual system. Due to the masked CCD rows the physical size of the spatial resolution element is $22.5 \mu \mathrm{m}$ in one direction and $90 \mu \mathrm{m}$ in the orthogonal direction. This large and asymmetric resolution element is poorly adapted to the requirements of imaging at highest spatial resolution, since it requires strong magnification (typically a factor of 5) from the telescope focus onto the CCD, leading to very long optical path lengths and keeping the risk of introducing optical aberrations. In highest spatial resolution polarimetry, CCDs with small pixels are used. Since near the diffraction limit the polarimetric sensitivity is not of highest priority, more classical polarimetric set-ups employing slow modulation in combination with polarising beam-splitters (dual beam polarimetry) is the way of choice. The only existing polarimeter working also in the near UV is the POLIS intrument (Schmidt et al. 2001), which offers the possibility of simultaneous spectropolarimetry in Fe I $6302 \AA$ and Ca II H $3968 \AA$ with highest spatial resolution.

\subsubsection{Temporal resolution}

If polarimetric sensitivity is not of highest priority, ZIMPOL II can be used in single frame mode. Since the modulation frequency is in the $\mathrm{kHz}$ regime, any short exposure will contain the full polarisation information provided by the modulator package. That means that - in the actual system based on a photoelastic modulator - Stokes $I$, Stokes $Q / I$, and 
Stokes $V / I$ are completely and cospatially recorded in a single exposure of, for instance, $20 \mathrm{~ms}$. This makes ZIMPOL II the primary polarimeter to be used in speckle or phase diversity applications.

\subsection{Outlook}

Although already a very useful instrument, the scientific value of ZIMPOL II could still be dramatically increased by a full vector modulation scheme. As indicated earlier, Pockels cells provide the necessary wave-forms and can be driven at $\mathrm{kHz}$ rates. At least at wavelengths above $350 \mathrm{~nm}$ a system based on two Pockels cells would be the primary choice of a modulator package for ZIMPOL. Such a system is currently under development at ETH Zurich. First tests are expected to take place in the near future.

Another drawback of the ZIMPOL system is the poor geometric filling factor of the CCD. This is on the one hand due to the mask, on the other hand it is related to the open electrode structure. The overall photon sensitivity could be drastically improved by the use of a microlens array that focuses the photons, which would normally fall on four pixels, onto the photosensitive area of the unmasked pixel. Such a microlens array has been manufactured for the visible versions of ZIMPOL II (Gale et al. 1997; Gandorfer \& Povel 1997). For the UV part, a new development has just been started. With these two improvements, ZIMPOL II will keep up with the development of new solar telescopes opening new opportunities for observations of the Sun.

Acknowledgements. The present work has been supported by the Swiss Nationalfonds, grant No. 20-64945.01. The IRSOL facility gratefully acknowledges financial support from the canton of Ticino, the city of Locarno, ETH Zurich and the Swiss Nationalfond. The McMath-Pierce facility of the National Solar Observatory is operated by the Association of Universities for Research in Astronomy, Inc. (AURA) under cooperative agreement with the National Science Foundation. The New Swedish Solar Telescope is operated by the Royal Swedish Academy of Sciences.

\section{References}

Bernasconi, P. N. 1997, Ph.D. Thesis, ETH Zürich

Bianda, M., Solanki, S. K., \& Stenflo, J. O. 1998a, A\&A, 331, 760

Bianda, M., Stenflo, J. O., Gandorfer, A., \& Gisler, D. 2003, in Current Theoretical Models and Future High Resolution Solar Observations: Preparing for ATST, ed. A. A. Pevtsov, \& H. Uitenbroek, ASP Conf. Ser., 286, 61

Bianda, M., Stenflo, J. O., \& Solanki, S. K. 1998b, A\&A, 337, 565

Bianda, M., Stenflo, J. O., \& Solanki, S. K. 1999a, A\&A, 350, 1060
Bianda, M., Stenflo, J. O., \& Solanki, S. K. 1999b, in Solar polarisation, Proc. 2nd Solar polarisation Workshop, ed. K. N. Nagendra, \& J. O. Stenflo (Dordrecht: Kluwer), 31

Gale, M. T., Pedersen, J., Schütz, H., et al. 1997, Optical Engineering, 36,1510

Gandorfer, A. M. 1999a, Optical Engineering, 38, 1402

Gandorfer, A. M. 1999b, in Solar polarisation, Proc. 2nd Solar polarisation Workshop, ed. K. N. Nagendra, \& J. O. Stenflo (Dordrecht: Kluwer), 297

Gandorfer, A. M. 2000, The Second Solar Spectrum, A high spectral resolution polarimetric survey of scattering polarisation at the solar limb in graphical representation, Vol. 1: $4625 \AA$ to $6995 \AA$ A (Zurich: vdf, ISBN 372812764 7)

Gandorfer, A. M. 2002, The Second Solar Spectrum, A high spectral resolution polarimetric survey of scattering polarisation at the solar limb in graphical representation, Vol. 2: $3910 \AA$ A to $4630 \AA$ A (Zurich: vdf, ISBN 372812844 4)

Gandorfer, A. M. 2004, The Second Solar Spectrum, A high spectral resolution polarimetric survey of scattering polarisation at the solar limb in graphical representation, Vol. 3: $3160 \AA$ to $3920 \AA$, in preparation

Gandorfer, A. M., \& Povel, H. P. 1997, A\&A, 328, 381

Gisler, D. 2004, Ph.D. Thesis, ETH Zurich, in preparation

Ivanov, V. V. 1991, in Stellar Atmospheres - Beyond Classical Models, Crivellari, Proc. NATO, ed. L. Crivellari, I. Hubeny, \& D. G. Hummer (Dordrecht: Kluwer), 81

Keller, C. U. 1996, Sol. Phys., 164, 243

Martinez Pillet, V., Garcia Lopez, R. J., del Toro Iniesta, J. C., et al. 1990, ApJ, 361, L81

Povel, H. 1995, Optical Engineering, 34, 1870

Povel, H., Aebersold, H., \& Stenflo, J. O. 1990, Appl. Opt., 29, 1186

Povel, H. P. 2001, in Magnetic Fields Across the Hertzsprung-Russell Diagram, ed. G. Mathys, S. Solanki, \& D. Wickramasinghe, ASP Conf. Ser., 248, 543

Povel, H. P., Keller, C. U., \& Yadigaroglu, I.-A. 1994, Appl. Opt., 33, 4254

Schmidt, W., Kentischer, T., Bruls, J., \& Lites, B. 2001, in Advanced Solar Polarimetry - Theory, Observation, and Instrumentation, ed. M. Sigwarth, ASP Conf. Ser., 236, 49

Stenflo, J. O. 1974, Sol. Phys., 37, 31

Stenflo, J. O., Baur, T. G., \& Elmore, D. F. 1980, A\&A, 84, 60

Stenflo, J. O., Keller, C. U., \& Povel, H. P. 1992, LEST Foundation Technical Report, 54

Stenflo, J. O., \& Keller, C. U. 1996, Nature, 382, 588

Stenflo, J. O., \& Keller, C. U. 1997, A\&A, 321, 927

Stenflo, J. O., Keller, C. U., \& Gandorfer, A. 1998, A\&A, 329, 319

Stenflo, J. O., Gandorfer, A., \& Keller, C. U. 2000a, A\&A, 355, 781

Stenflo, J. O., Keller, C. U., \& Gandorfer, A. 2000b, A\&A, 355, 789

Stenflo, J. O., Gandorfer, A., Wenzler, T., \& Keller, C. U. 2001, A\&A, 367,1033

Wiehr, E. 1975, A\&A, 38, 303

Wiehr, E. 1978, A\&A, 67, 257 


\section{Online Material}




\section{Appendix A: System overview}

Figure A.1 presents a block diagram of the ZIMPOL II system. Hardware and software are described in more detail in the following sections. The dashed blocks are not part of ZIMPOL II. Light from the telescope passes through the Calibation Optics and the Modulator Package, before it enters the wavelength discriminator, which can be either a spectrograph or any type of (tunable) filter. The demodulating CCD camera is placed at the end of the optical train. Control of the hardware and image processing is performed by the Control and Image Processing System (CIPS). The system-wide Hardware Control Network (HCN) transmits data and commands to and from the different hardware units distributed in the system. For details on the HCN see Appendix C. The CIPS is connected to local and remote workstations and notebooks via Ethernet.

\section{Appendix B: Polarisation modulation}

The modulator package consists of one photoelastic modulator (PEM) followed by a Glan linear polarizer. With this setup the circular polarisation is modulated at the mechanical vibration frequency of the modulator, which is in our setup $42 \mathrm{kHz}$, while one component of the linear polarisation is modulated at twice this frequency, thus $84 \mathrm{kHz}$. The other component of the linear polarisation is not modulated. The modulation system is described in detail in Gandorfer \& Povel (1997).

We use a PEM-90 model by Hinds Inc. with a useful aperture up to $63 \mathrm{~mm}$ and a mechanical vibration frequency of $42 \mathrm{kHz}$. The modulator head is made from fused silica and is multilayer coated to avoid internal reflections between $300 \mathrm{~nm}$ and $600 \mathrm{~nm}$. For higher wavelengths another PEM optimized for $500 \mathrm{~nm}$ to $800 \mathrm{~nm}$ can be used.

The PEM periodically modulates the incoming polarisation. This polarisation modulation is converted into a periodic intensity modulation via a linear polarizer which acts as an analyzer. The linear polarizer is a Glan prism made of calcite being transparent between $220 \mathrm{~nm}$ and $2.2 \mu \mathrm{m}$, made by Halle, Berlin, with a useful aperture of $44 \mathrm{~mm}$.

As this setup does not allow for simultaneous detection of Stokes $Q$ and $U$, the whole modulator package can be rotated. In this way Stokes $I, Q / I$ and $V / I$ is measured with one setting of the modulator package, while $I, U / I$ and $V / I$ is measured in a second exposure with the modulator package being rotated by $45^{\circ}$. Stokes $I$ and $V / I$ are thus detected in both exposures, while the components of the linear polarisation are detected alternately.

Simultaneous detection of the complete Stokes vector in a single CCD exposure is currently not possible in the UV range, since it requires a four-fold, fast, electrooptical modulation scheme. Different solutions are theoretically thinkable, like the use of a phase-stabilized pair of photoelastic modulators, as proposed initially for the use in the ZIMPOL II polarimeter by Stenflo et al. (1992). A first prototype of such a Dual PEM has, however, failed to ensure the extreme stability requirements needed for high precision polarimetry. Another solution would be the use of a pair of Pockels cells, which would allow for ideal modulation, and would also be wavelength tun- able. However, below $350 \mathrm{~nm}$, absorption takes place in the "transparent" ITO electrodes, which are deposited on the optical faces of the Pockels cell. Still, the promising advantages of a pair of Pockels cells justify a technology development program with industry, which is currently under way.

\section{Appendix C: CCD sensor}

A drawing of the geometry of the new sensor, showing pixel and electrode sizes, and the position of the aluminium mask is presented in Fig. C.1. UV-enhancement is achieved by etching holes into poly-silicon layers 2 and 3. To increase the sensitive area the 3 shielded lines have been reduced in height, allowing greater height for the open line. Figure C.1 shows the structure of a modified CCD55-20 with the height increased to $27 \mu \mathrm{m}$ for the open line and reduced to $21 \mu \mathrm{m}$ for each of the other lines, giving a total height of $90 \mu \mathrm{m}$ of the 4-pixel block. Due to structural restrictions (certain feature sizes cannot be reduced arbitrarily), and the requirements of a metal shield as well as fast bi-directional vertical charge transfer, the greatest open area which can be made is $12.5 \mu \mathrm{m} \times 12.5 \mu \mathrm{m}$, which is $\approx 26 \%$ of the total pixel area. Further increases in line height would increase the length of the transfer path around the aperture with a consequent loss of vertical transfer speed, which is not tolerable with ZIMPOL II. The metal shield covering 3 out of 4 lines has an aperture of $22.5 \mu \mathrm{m}$.

With respect to transfer efficiency and maximum charge transport operation of the new device at fast vertical transfer proved to be more critical than of the older CCD05-20. The most critical parameter is the vertical transfer time $\tau$ which has to be much shorter than the modulation period. $\tau$ is limited by the capacitance and the resistance of the image area electrodes, which determine the intrinsic time constant of the distributed $\mathrm{RC}$ transmission lines inside the CCD chip. In addition $\tau$ is limited by high transient currents flowing during fast vertical clock transitions, and leading to considerable heating of the chip.

Another problem arises with charge traps which are known from CCD testing by a method called pocket pumping: by frequently shifting charge packets across a trap in both directions, the depth of the trap is measured. With ZIMPOL II this effect shows up during demodulation, when the chip is exposed to unpolarized light and the trap is gradually filled and adjacent pixels become depleted. The size of the traps depends to some extend on the transfer speed, but it also depends on chip parameters, which cannot be controlled outside the factory. Traps appear as large randomly distributed peaks (relative difference between adjacent pixels) in the demodulated signal, obtained with unmodulated light. They increase with integration time, and finally limit the integration time.

With ZIMPOL I a Marconi CCD02-06 $(2 \times 288(V) \times$ $385(H)$ pixels, $22 \mu \mathrm{m} \times 22 \mu \mathrm{m})$ is used. It is operated at $\tau=$ $600 \mathrm{~ns}$. This allows demodulation at $100 \mathrm{kHz}$ in combination with a small PEM-80 (Hinds) modulating linearly polarised light (2f mode). The four times larger CCD55-20 used with ZIMPOL II is operated at $\tau=1.5 \mu \mathrm{s}$, which is at the very limit of what can be achieved with this CCD. This allows demodulation at $84 \mathrm{kHz}$ with a Hinds PEM-90 in $2 \mathrm{f}$ mode. 
A. M. Gandorfer et al.: Near UV polarimetry, Online Material p 3

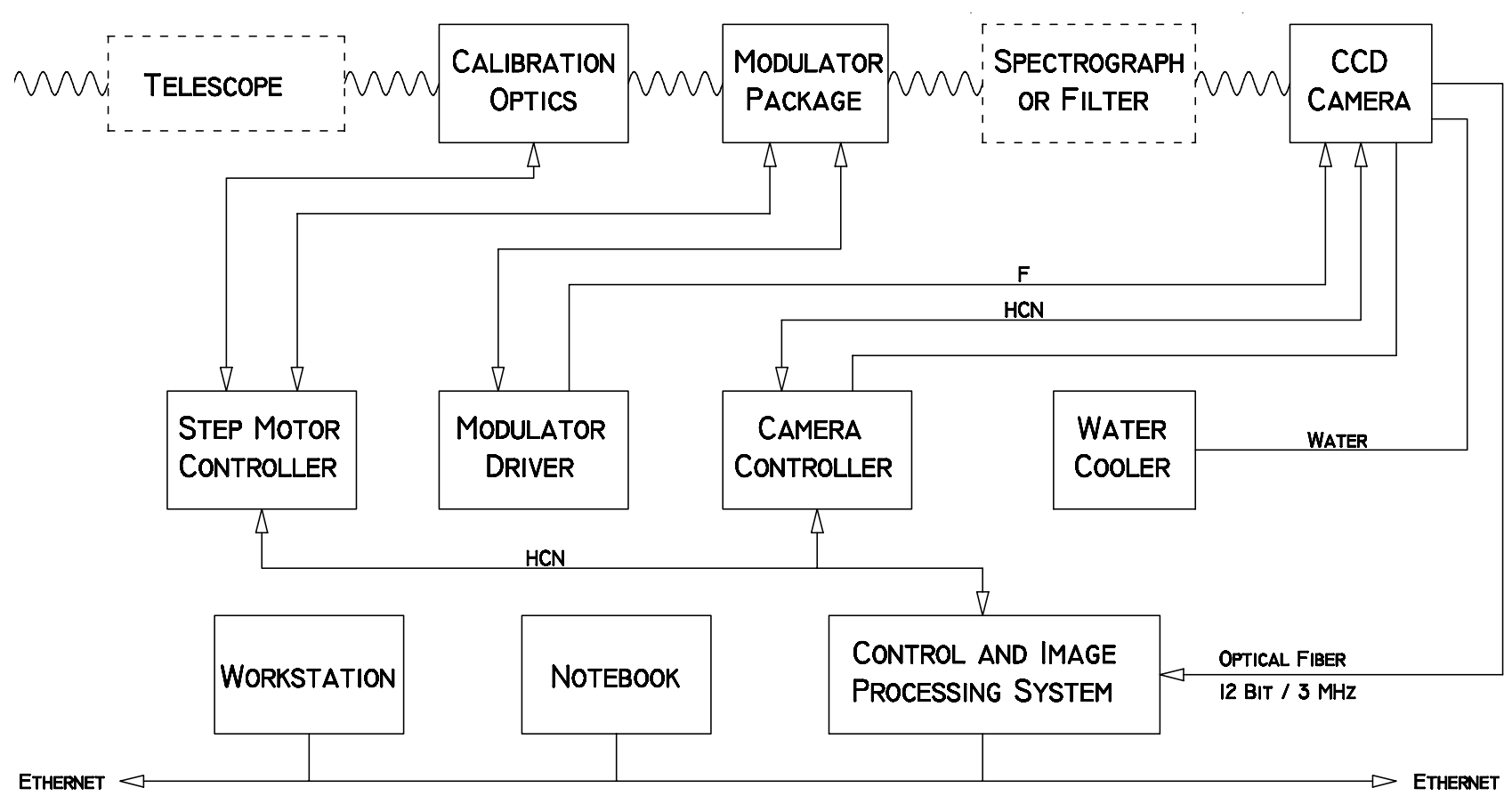

Fig. A.1. Block diagram of the ZIMPOL II system. Connections not labeled indicate diverse power and control lines. HCN indicates the Hardware Control Network, F the reference signal for the demodulator. The dashed blocks are not part of ZIMPOL II.

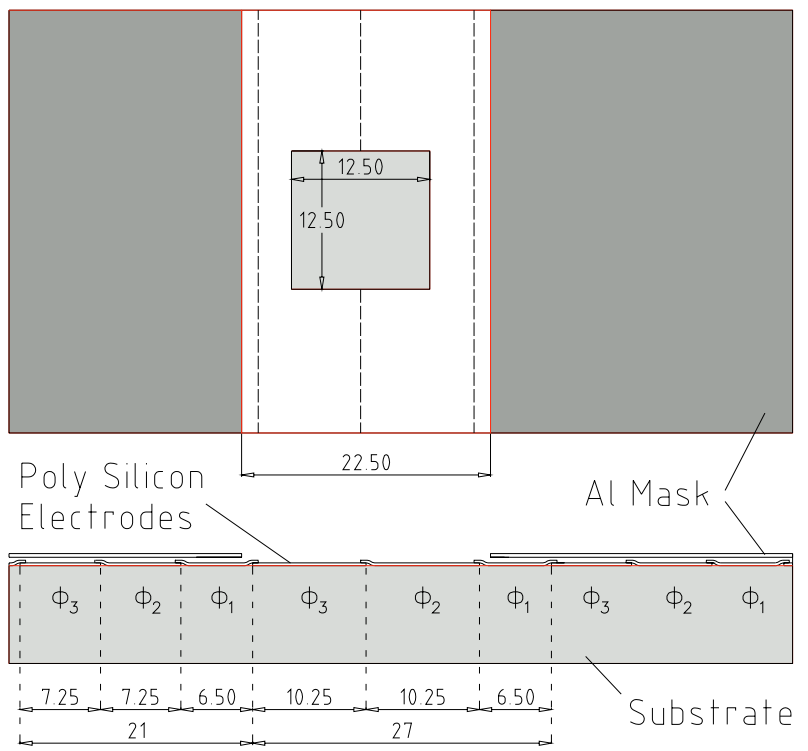

Fig. C.1. Open electrode structure of masked UV-enhanced Marconi CCD55-20. $\Phi_{1}, \Phi_{2}, \Phi_{3}$ indicate the three electrodes defining one pixel. All measures are given in $\mu \mathrm{m}$. Bottom: cross section in column direction. Top: Front view with open electrodes, and Al mask. The center to center distance between the holes is $22.5 \mu \mathrm{m}$ in row direction and $(3 \times 21+27) \mu \mathrm{m}=90 \mu \mathrm{m}$ in column direction.

\section{Appendix D: CCD camera}

The camera consists of two units: the evacuated head and attached to it the electronics box. The head contains the CCD, a two-stage thermo-electric cooler (TEC), and a printed circuit board (PCB) with ZIF socket for the CCD, preamplifiers, decoupling networks for the CCD voltages, and a driver for the CCD reset pulse. The head has a diameter of $136 \mathrm{~mm}$. Its frontside is provided with a $60 \mathrm{~mm}$ diameter quartz window with anti-reflective coating optimized for $\mathrm{UV}$, and $0.5^{\circ}$ wedge angle. To prevent frosting of the CCD chip, the housing is evacuated to about $0.1 \mathrm{mbar}$. Due to leakage of the order of $0.5 \mathrm{mbar}$ per day it has to be pumped after 2 to 4 months. Water evaporating from components inside the housing and from its surface is adsorbed by molecular sieve filled in a cavity at the back plate. A two-stage TEC (Marlow MI 2063T) is directly mounted on the back plate, which is provided with channels for cooling water needed to remove the thermal energy dissipated by the TEC. The cooling water is circulated by a pump and cooled by a second TEC. At $80 \mathrm{kHz}$ demodulation with PEMs a temperature difference of $-50^{\circ}$ between cold and hot side of the TEC can be maintained with still $20 \%$ reserve. The cold side of the TEC is coupled to the CCD via a $12 \mathrm{~mm}$ spacer. Temperatures are measured at both sides of the TEC with Pt100 elements. The PCB is attached to the back plate, and connected to the outside via two 26-pin feedthrough header connectors. The connectors are glued into the back plate.

\section{D.1. CCD Camera electronics}

The electronics box of the CCD camera head contains four $200 \mathrm{~mm} \times 160 \mathrm{~mm}$ PCBs, plugged onto a back plane mounted on the rear side of the head. The back plane connects the PCBs among each other and to the CCD and electronic components located inside the head. The drivers for the CCD phase pulses are placed on the back plane. On the first board signal processing components like amplifiers, CDS processor and ADC are located. The second board is occupied by regulators supplying different voltages to the CCD and the electronic components. The third board accomodates a fiber optical link, based on a TAXI chip, for transmission of the 12-bit pixel data, a 


\section{A. M. Gandorfer et al.: Near UV polarimetry, Online Material p 4}

$\mathrm{HCN}$ interface, and two interfaces for the Pt100 temperature sensors including ADCs. The system is laid out for a two-port $\mathrm{CCD}$ with maximum $2 \times 2.5 \mathrm{MHz}$ 2-byte pixel rate corresponding to a $10 \mathrm{MByte} / \mathrm{s}$ data flow. The third board is occupied by digital electronics needed to control the camera: sequencer for the CCD 3-phase clock pulses, timing of CCD clocks, readout and signal processing, integration timing, demodulation mode and phase. Temperature data as well as parameters and commands, needed to control the camera functions are transmitted via the $\mathrm{HCN}$.

The camera is connected to the Camera Controller, which forwards HCN informations from and to the Control and Image Processing System (CIPS), and contains several power supplies for the camera electronics and the TEC. Pixel data and frame informations are transmitted to the CIPS via a fiber optical link. The reference signal, needed for the demodulation process is delivered by the modulator controller. All control lines connected to the camera are optically isolated.

\section{Appendix E: Step motor controller}

Depending on the optical setup up to 10 step motors are needed to rotate and move optical components in the ZIMPOL II modulator and calibration packages. The controllers can drive 2-phase step motors with currents up to 2 A per phase with 400 half steps per rotation, at step frequencies up to $4 \mathrm{kHz}$, or in micro-step mode with up to 6400 steps per revolution. They allow to drive several motors synchronously with the same clock. This feature is needed at certain solar telescopes where the image rotates during observation. Commands and parameters needed to control the motors, as well as signals from position sensors are transmitted to and from the CIPS via the HCN.

\section{Appendix F: Hardware control network}

The HCN is a simple interconnection of the CIPS with the various control circuits implemented in FPGAs and located in different hardware units. The HCN is designed to support the transfer of commands or status events and parameters requiring few wires and minimal circuitry in the FPGAs. Its topology is a star and data transmission is synchronous serial. The CIPS interface to the $\mathrm{HCN}$ is the master, provides the clock signal, and has the built-in capability to poll for input events and long latency input parameters. Output and non polling input transfers have low latency. Multiplexers and end points are non blocking and in their basic form require only a shift register and little glue logic. They can be implemented in FPGAs, close to the target circuits, avoiding long internal parallel busses. Multiplexers consume the address bits they interprete in the downstream. End nodes respond immediately at least by an acknowledegement of the request and optionally with a ready flag and a response.

\section{Appendix G: Software}

The main purpose of the ZIMPOL II software is to coordinate control of the various subsystems (camera, modulator, calibra- tion package, etc.), acquire frames of image data from the camera, preprocess and display them in real-time, reduce and store them to disk files, and perform simple statistics. Preprocessing includes a dark field correction, the application of the demodulation matrix to calculate the four Stokes components, and a flat field correction of the intensity image. Data reduction is based on simple summing, and in a future extension might include frame selection.

The ZIMPOL II software has to provide both a graphical and a command line user interface, and support command scripts. The user interfaces should be portable to any Notebooks or workstations connected to the CIPS via Ethernet and Internet, and should allow for a concurrent use, e.g. remote assistance etc.

The image processing should be able to accept more than 5 frames per seconds in bursts, and more than 2 frames per second in continuous mode with a reasonable reduction.

\section{G.1. Concepts}

The ZIMPOL II software has been designed as a distributed system that allows to run some components on the CIPS and others on Notebooks or workstations connected to it through the Internet. The distribution of tasks was considered necessary to releave the CIPS processor from the somewhat demanding tasks related to the user interfaces. It has additional advantages that are mentioned below.

The distribution of tasks leads to a strict separation between programs that perform basic functions (like coordination of control, image acquisition, preprocessing, and reduction) and others that provide graphical and command line interfaces or convert and store data as FITS files. Since the basic functions are bound to the CIPS while the others may run anywhere, the two types of programs must be able to communicate through the network, e.g. TCP stream sockets, for both control and data streams. Separating basic tasks from user interface programs also opens the way for concurrent use of multiple user interfaces, multiple realtime displays, assisted observation, remote observation, etc.

The programs that implement basic tasks have different properties and execution characteristics than those that realize user interfaces. The former are bound to the image input and hardware controller interfaces of the CIPS, have more predictable and static needs for resources (processor, memory, communication, files), may cause a high processor utiliziation, can be optimized with respect to performance and memory access patterns, use multithreading to realize loosely coupled concurrent subtasks, and must run as exactly one instance during the entire observation session. The latter, in particular the graphical interface programs, have more unpredictable and dynamic needs for resources, are organized around the X-windows event loop, share window-related services that cause response times to vary, utilize the processor in short bursts, may run in multiple instances typically on different machines, and may be started and stopped during an observation session. The separation of user interface tasks from basic func- 


\section{A. M. Gandorfer et al.: Near UV polarimetry, Online Material p 5}

tions allows to keep both sides simple and free from the typical constraints of the other.

Removing these user interface tasks from the CIPS processor simplifies resource utilization pattern on it and makes optimiziation of the basic task more effective. It typically also may reduce network traffic to display images (via $\mathrm{X}$ server) and store image data (through NFS) by a factor of 2 to 3, depending on the configuration. The outlined distribution of tasks thus also improves the conditions for using a general purpose processor inside the CIPS.

Despite the distribution and separation of tasks, the basic functions, if implemented in software as processes and threads under a Unix operating system, cannot really meet hard realtime requirements. Fast or low-latency $(\leq \mathrm{ms})$ control operations had to be realized in hardware, i.e. in FPGAs located in the various units. The image input had to be FIFO-buffered $(64 \mathrm{kB})$ to hide DMA driver interrupt latency. To tolerate the somewhat stochastic latency of the functions realized in software, the entire system including communication must strictly be made event-driven, where events include both data records or free resources like buffers becoming available. This garantees that the result is correct under all timing conditions and any set of limited resources, and it lets the system run as fast as the slowest component of a particular configuration allows for.

\section{G.2. Implementation}

The basic functions have been implemented as two processes, both multithreaded, one dedicated to image preprocessing (IPP), the other to collect, serialize and interprete command streams, coordinate control, and broadcast state changes (CSI).

To achieve acceptable performance, a fixed scheme of preprocessing and reduction is being used, which avoids unnecessary copy operations and memory reallocations, and helps exploiting caching and cache prefetching. Processing time can be reduced by choosing smaller regions of interest for display or reduction, or by reducing update rates of displays or statistics, etc. A large input buffer allows for higher speeds during bursts of up to 60 frames by decoupling frame acquisition, preprocessing, and data reduction from each other. All image buffers are memory-mapped files that can be accessed for test purposes.

The graphical user interface (GUI) is realized as two programs, one for control and the other for image data display, because of the different data source and time characteristics. Both are necessarily organized around the X-windows event loop. They are single-threaded but react to events both from graphical input and CIPS response. They handle commands and responses asynchronously with respect to each other. The relevant state of the control GUI is maintained by the common command stream interpreter (CSI) running on the CIPS. Commands are being serialized by the CSI. The CSI brodcasts parameter changes and status information to all connected GUIs. The GUI uses colors to distinguish parameter changes as a response to a local entry from those caused by a remote GUI or the execution of a script. All GUI action routines (that respond to events), are being serialized but have execution times not conceivable by the user in typical conditions.

The CSI can serve multiple GUIs and other command clients, that may also send scripts. Per default, commands are executed sequentially. The CSI provides constructs to execute statements concurrently, in the background, or in the foreground. The execution of these statements appears to be in parallel if they involve commands that include waiting for some timed or external event like the end of the exposure or a mechanical movement. This kind of parallelism is useful to setup multiple mechanical devices, to interrupt or supervise sequential execution, and for changing the optical setup for every acquired frame in single step mode, etc.

The CSI controls the IPP and the hardware units through a bidirectional downward control stream. More such connections to other subsystems may be established dynamically. The CSI provides the notion of channels and associated parameters and commands or status events. It also supports a kind of parameter transformation routines that may perform simple calculations or bookkeeping actions before a parameter is being sent or a response is being referenced.

Data streams and control streams between ZIMPOL II programs are all realized as TCP stream connections (using stream sockets). The messages sent over control streams in any direction between the CSI and the IPP and between the GUIs and the CSI all share the same simple and common general plaintext (ASCII) format. The format syntactically distinguished parameter setups or changes, parameterless commands, and status information. Parameters are global and precede commands that use them. Programs that interprete some of the messages do not need to know the definitions of the others to be able to forward them. Down- and upstreams are not interlocked but rather asynchronous to each other. Requests (commands or parameter setups) and responses (status or parameter changes) are interrelated only by the client and server processes rather than by the commmunication protocol. The protocol allows thus a client to control concurrent actions in a server if the latter supports concurrency.

The CSI does not distinguish parameters from variables syntactically. It even provides the same uniform syntax for references to parameter transformation functions and procedures. However, an assignment to a parameter results in a parameter setup being sent to a subsystem like a hardware controller, while an assignment to a variable has no external effect. The CSI maintains shadow variables for write-only parameters. Statements that contain references to parameters or external commands must be decomposed stepwise by the CSI into atomic parts that can either be executed immediately or else depend on exactly one external event. An external command or a parameter setup is considered to complete only after the confirming event has been received. The CSI maintains an event wait queue for each channel to temporarily hold such commands until the expected response is being received. By decomposing statements and maintaining queues the CSI can handle concurrent statements without ever blocking itself. It starts the evaluation of a subexpression only after all operands have been made available internally. 


\section{A. M. Gandorfer et al.: Near UV polarimetry, Online Material p 6}

IPP and CSI are written in C and use multithreading under Digital Unix (OSF/1). The GUIs are written in $\mathrm{C}$ and originally based on OSF/Motif using descriptor methids including UIL. Alternative but somewhat experimental versions have been realized under Borland Delphi for Windows and later in Java for any platform. Command line interfaces and other utilities are also written in $\mathrm{C}$ for a more portable POSIX environment.

\section{Appendix H: Calibration optics}

The complete polarimeter can be exposed to light of known polarisation states. A combination of a linear polariser and an achromatic quarter-wave plate can be remotely inserted into the beam in front of the modulator package. Both components can be remotely rotated and thus any state of polarisation can be created. To cover the whole useful wavelength range of the instrument several polarizers are needed: A dielectric beam-splitter in the form of a stretched cube by Halle (Berlin) with $40 \mathrm{~mm} \times 40 \mathrm{~mm}$ square aperture and $56 \mathrm{~mm}$ length is used as linear polariser for the wavelength range from $300 \mathrm{~nm}$ to $410 \mathrm{~nm}$. A second dielectric beam-splitter cube (also from Halle) with the same dimensions covers the range from $390 \mathrm{~nm}$ to $500 \mathrm{~nm}$. For the visible part of the spectrum common dielectric beam splitter cubes are employed, useful in the wavelength ranges from $440 \mathrm{~nm}$ to $650 \mathrm{~nm}$, and $600 \mathrm{~nm}$ to $900 \mathrm{~nm}$, respectively.

The retardation plates for the different wavelength ranges are all manufactured by Halle (Berlin) in the form of achromatic combinations of quartz and $\mathrm{MgF}_{2}$. One plate is optimised for the range from $300 \mathrm{~nm}$ to $470 \mathrm{~nm}$; above this range similar plates are employed, one for the range from $460 \mathrm{~nm}$ to $680 \mathrm{~nm}$, another one for the range from $600 \mathrm{~nm}$ to $1200 \mathrm{~nm}$.

All components are mounted onto motorized rotation stages (OWIS DMT 100) which allow free rotation of the components. The rotation stages are mounted onto precision motorized linear positioning units (THK KR 20) which allows the calibration optics to be remotely inserted into and removed out of the light beam.

\section{Appendix I: Optical layout}

The ZIMPOL II CCD camera has $770 \times 576 / 4$ pixels with $22.5 \mu \mathrm{m} \times 90 \mu \mathrm{m}$ effective pixels. In spectropolarimetric applications it can be illuminated through telecentric reduction optics to adapt the linear dispersion of solar spectrographs to an appropriate value of typically $10 \mathrm{~m} \AA$ per pixel. For this a linear reduction factor of 2.75 was choosen. The optical setup is a Keplerian telescope with a field lens in the spectrograph focal plane which provides a real image of the camera mirror. This image is imaged to infinity by the second reduction lens which at the same time reimages the field lens main plane and thus the spectrum onto the CCD. After the reduction lens the principle image rays are parallel (telecentric configuration) and the CCD pixels all are affected in the same way by illumination effects like f-ratio limitations or field of view effects.

The field lens is a three lens UV apochromat with $300 \mathrm{~mm}$ focal length and $60 \mathrm{~mm}$ free aperture. As reduction lens an aplanatic design has been choosen, made from two identical air spaced UV achromatic doublets in a symmetrical configuration. The focal length of the individual achromats is $200 \mathrm{~mm}$. With a separation of their back cardinal planes by $30 \mathrm{~mm}$ a resulting focal length of $108 \mathrm{~mm}$ is achieved thus leading in combination with the $300 \mathrm{~mm}$ field lens to the desired reduction factor of 2.75. All lenses are made from crystalline quartz or $\mathrm{CaF}_{2}$ and are transparent between $200 \mathrm{~nm}$ and $2.5 \mu \mathrm{m}$. The apochromatic correction is good in the whole useful wavelength range of the polarimeter, from the atmospheric cut-off around $300 \mathrm{~nm}$ to the silicon band gap limit around $1.1 \mu \mathrm{m}$. Since no adequate antireflection coating is available to cover the whole wavelength range we use a multilayer AR coating optimised for the wavelength interval between $300 \mathrm{~nm}$ and $600 \mathrm{~nm}$.

\section{Appendix J: Calibration procedure and observational technique}

\section{J.1. Vectorpolarimetry below $4000 \AA$}

Since below $4000 \AA$ a single piezoelastic modulator is used, the modulation package must be rotated to alternate between a recording of Stokes $I, Q / I$, and $V / I$, and a recording of $I, U / I$, and $V / I$, as described in Appendix B.

This method is suitable if the total integration time is much longer than needed to rotate the modulator package or than the exposure time per frame. This is the case for observations of weak polarisation signals for example the detection of scattering polarisation at the solar limb, which - with current solar telescopes - requires total integration times around 10 to $20 \mathrm{~min}$.

\section{J.2. Calibration procedure}

\section{J.2.1. Dark frame exposition}

All CCD data must be corrected for bias and dark current. Both bias and dark current constitute the dark frame. While the bias is common to all pixels, thermally induced charges are individual to every pixel; the dark frame is therefore subtracted pixel by pixel from the measurement frames. Since the total signal of the dark frames is low, also the standard deviation of the dark frame is low as compared to the measurement frames, which are dominated by their photon statistics. For high precision polarimetric measurements dark-current and bias must be very stable. The influence of bias drifts and dark-current inaccuracies is discussed by Keller (1996). Any temperature change will not only lead to dark-current errors but also affect the read-out capacity leading to bias and gain changes, which is the more severe effect. The high electric current through the ZIMPOL II gate electrodes, which is a consequence of the rapid periodic change of the applied potentials, poses high demands on the control of the CCD temperature. In order to keep the CCD temperature as stable as possible, the CCD is always driven in the same way as during the measurement, even if the CCD is not used for measurements for a certain time. This keeps the electric and thus the thermal environment stable enough to ensure the necessary temperature stability. 


\section{A. M. Gandorfer et al.: Near UV polarimetry, Online Material $p 7$}

\section{J.2.2. Polarimetric calibration}

Polarimetric calibration of the instrument is done in front of the polarimetric analysis package, but normally after oblique reflections that occur in most solar telescopes. Therefore, in the following section polarimetric calibration means only the calibration of the efficiency matrix of the polarimeter, not taking into account the Mueller matrix of the telescope. The calibration procedure has been developed quite early by Bernasconi (1997) for the ZIMPOL I polarimeter. Since the working principles of the UV sensitive version of ZIMPOL II and ZIMPOL I are basically equivalent, the calibration procedure is also valid for our new polarimeter. The instrument's polarimetric efficiency for Stokes $Q$ is calibrated by inserting a linear polariser into the light beam in the positive Stokes $Q$ position (which is defined to be the exit polarisation direction of the modulator package in it's default position; this is normally parallel to the entrance slit of the spectrograph). To correct for the variation of the polarimetric efficiency across the photoelastic modulator (caused by the excited standing acoustic wave), an additional measurement with small linear polarisation is required, as described in detail in Bernasconi (1997) or in Gandorfer (2000). The necessary amount of linear polarisation is in most cases produced in the telescope by oblique reflections. At telescopes with very small instrumental polarisation a tilted glass plate can be used. The efficiency of the instrument to Stokes $V$ is measured by inserting a quaterwave plate in addition to the linear polariser. Since an observation of Stokes $U$ requires the rotation of the modulator package by $45^{\circ}$ one could consider using the same calibration measurement as used for Stokes $Q$. The retardance pattern in the PEM is, however, not rotational symmetric, such that an individual calibration for Stokes $U$ is required.

\section{J.2.3. Calibration for charge traps}

For most pixels the charge transfer inefficiency (relative amount of charge which is lost during a single pixel shift) is negligible. In the EEV CCD 02-06 employed in the ZIMPOL II prototype is has been measured to be as low as $2 \times 10^{-8}$ per shift (Gandorfer \& Povel 1997). As a consequence of the large amount of bidirectional charge shifts on the CCD, asymmetries in the shift process leads to incorrect polarisation signals. Three different effects are known: (1) A timing asymmetry in the demodulation cycle causes a constant offset in the demodulated difference images. (2) The charge transfer inefficiency is slightly different for both transfer directions. The magnitude of this asymmetry is individual for all pixels. Thus every pixel quadruple (group of 4 neighbouring pixels forming one demodulation group) produces a small amount of erroneous difference signal, which induces the socalled "fixed pattern noise" in the demodulated images. Without calibration this noise is on the order of $10^{-3}$. (3) Individual pixels with crystal defects may act as socalled "charge pockets" or "charge traps". The asymmetry in the charge transfer inefficiency of these pixels is anomalously large. These pixels are in most cases isolated, only in rare cases pairs or clusters of pixels are found. Although depending nonlinearily on intensity and integration time, in most cases the error signal is reproducible, which allows at least for partial compensation. Two techniques can be applied:

\section{Synchronous/asynchronous demodulation}

Synchronous and asynchronous demodulation are two different measuring modes of ZIMPOL II. In the synchronous mode the demodulation is synchronous in frequency and phase to the modulation of the PEM. It is the normal measuring mode, which is sensitive to the polarisation of the incoming light. In the asynchronous mode the synchronisation between PEM and CCD is switched off, therefore the system is blind to polarisation. However, all spurious effects related to the charge shifting scheme itself are still detected. If one takes an exposure in the synchronous mode and one in the asynchronous mode, which is taken under the same observing conditions, then the difference between the two images will be free from all spurious charge shifting effects. There are two disadvantages of this technique: the demodulation frequencies in the two modes must be slightly different, thus the amount of the spurious effect may not be exactly the same and cannot be fully compensated. Since the asynchronous exposure does not contain information on the real polarisation, the polarimetric efficiency is reduced by a factor of two, thus the effective noise level in the observation is increased by a factor of $\sqrt{2}$.

\section{Synchronous demodulation with different phases}

The sign and amplitude of the detected polarisation signal depends on the freely adjustable phase between modulation and demodulation. By adding a fixed phase the sign of the signal can be reversed. Since all spurious charge shifting effects are independent of the phase relation, the difference of the two exposures will yield twice the real polarisation value, while compensating the spurious error signals. Due to the fact that in the ZIMPOL II modulation scheme Stokes $Q$ and $V$ are simultanously modulated it is not possible to find two phase values, which give optimum polarimetric efficiency for both Stokes parameters. Thus the total polarimetric efficiency is reduced. 